\title{
BEYOND PROJECTION: \\ USING COLLABORATIVE VISUALIZATION TO CONDUCT QUALITATIVE INTERVIEWS
}

\author{
Alice Comi \\ Design Innovation Research Centre \\ University of Reading, UK \\ Nicole Bischof \\ Institute of Media and Communications Management \\ University of St. Gallen, Switzerland \\ Martin J. Eppler \\ Institute of Media and Communications Management \\ University of St. Gallen, Switzerland
}

This is the Authors' accepted manuscript for self-archiving purposes. Please refer to the final publication on Emerald's website for pages and citation. The final publication is available on: http://www.emeraldinsight.com/doi/abs/10.1108/QROM-05-2012-1074 
BEYOND PROJECTION:

USING COLLABORATIVE VISUALIZATION TO CONDUCT QUALITATIVE INTERVIEWS

\begin{abstract}
Purpose - This article argues for the reflective use of visual techniques in qualitative interviewing and suggests using visuals not only as projective techniques to elicit answers, but also as facilitation techniques throughout the interview process.

Design/methodology/approach - By reflecting on their own research projects in organization and management studies, the authors develop a practical approach to visual interviewing - making use of both projective and facilitation techniques. The article concludes by discussing the limitations of visualization techniques, and suggesting directions for future research on visually-enhanced interviewing.

Findings - The integration of projective and facilitation techniques enables the interviewer to build rapport with the respondent(s), and to elicit deeper answers by providing cognitive stimulation. In the course of the interview, such an integrative approach brings along further advantages, most notably focusing attention, maintaining interaction, and fostering the coconstruction of knowledge between the interviewer and the interviewee(s).

Originality/value - This article is reflective of what is currently occurring in the field of qualitative interviewing, and presents a practical approach for the integration of visual projection and facilitation in qualitative interviews.
\end{abstract}

Keywords Qualitative interviewing; one-to-one interviews; focus groups; visual techniques; projective techniques; visual representations; visual facilitation.

Paper type Research paper 


\section{BEYOND PROJECTION: \\ USING COLLABORATIVE VISUALIZATION TO CONDUCT QUALITATIVE \\ INTERVIEWS [1]}

\section{Introduction}

Despite increasing interest in visual techniques for qualitative interviewing (Davison, McLean and Warren 2012), a research methodology that exploits the full potential of the visual language is still largely missing. In this paper, we approach this gap and suggest using visuals not only as projective techniques to provoke or elicit comments from the respondents, but also as facilitation techniques to conduct interviews. We argue that the integration of projective and facilitation techniques will lead to the generation of richer data, as well as to greater involvement of research participants.

In projective techniques, visual stimuli are used to prompt reactions from the respondents, and to stimulate further thoughts on the interview topic (Crilly et al., 2006; Kearney and Hyle, 2004; Meyer, 1991; Vince and Broussine 1996; Zuboff 1998; Wheeldon 2011). In essence, projective techniques evoke reactions to a familiar stimulus (i.e., an image) in order to help participants elaborate their thoughts about a complex subject (Greenbaum, 2000). In the facilitation approach, by contrast, the interviewer uses visual templates for real-time documentation, organization, and discussion of the answers elicited from the respondents. A visual template combines the visual and verbal language by providing a graphical canvas where knowledge units - in the form of textual notations - are meaningfully structured, related, and put into perspective (Huff and Jenkins, 2002). As they are progressively filled out throughout the interview, visual templates become a repository of emergent knowledge, while also suggesting connections and revealing 'holes' in current information. In addition to fostering a shared understanding of the interview process, visual facilitation brings analytical advantages, since visual templates provide an organizing framework for analysing data.

With a view to leveraging the advantages of both projective and facilitation techniques, this paper proposes an integrative approach to visual interviewing where visuals are drawn, commented and interpreted in conversations between the interviewer and the participants. In this approach, the visual language becomes an integral component of the research process, being used first to elicit comments from participants (i.e., as projective techniques), and then to facilitate the interview process (i.e., as facilitation techniques). The participant is thus exposed 
to visual stimulation, takes part in the co-construction of the visual template, and finally can offer his or her interpretation of the completed graphic template. We have developed such an approach by reflecting on the extant literature on visual methods and by deriving first-hand insights from our own experience in organization and management research. As illustrated in the section "Examples from the Field", we have been using visuals for projective, facilitation, and analytical purposes in two research projects involving both interviews and focus groups. It was not the aim of these projects to formally test the validity of visual techniques in qualitative interviewing. Rather, we have adopted visual techniques to increase the breadth and scope of qualitative interviewing. The two projects were not designed specifically for this paper, but will be used here to illustrate our research experience and to reflect on the combined use of projective and facilitation techniques.

While visual techniques can be used on the ground of different epistemological and ontological assumptions (Davison et al. 2012), in our research we ascribe to a position of moderate constructivism. In this perspective, we assume that reality is apprehended through socially and experientially based mental constructions. The form and content of these constructions vary depending on the individual person or group holding them (Guba and Lincoln, 1994). While recognizing the relative nature of mental constructions, we refrain from the extreme assumption that such constructions have no independently identifiable real-world referents. Instead, we believe in the possibility of identifying the underlying patterns of a real-world phenomenon, by comparing and contrasting multiple individual constructions (Alvesson and Sköldberg, 2009). We therefore use visual techniques as a means to surface individual constructions in a closer collaboration with our informants, and we attempt to distil a "consensus construction" (Guba and Lincoln, 1994: 111) that approaches the phenomenon of interest in a more elaborate and informed way than any of the preceding constructions.

We structure our paper as follows: First, we review current attempts at using visuals as projective techniques in qualitative interviewing, and we point out the benefits as well as the limitations of such techniques. Subsequently, we argue for the introduction of visuals as facilitation techniques based on the idea of better exploiting the potential of the visual language and of enriching the range of visual methods for organization and management research. To this end, we discuss examples from our own research projects, and we develop a practical method for combining visual projection and facilitation in interviews. We conclude by describing the limitations of our approach and by suggesting directions for future research on the use of visual methods in qualitative interviewing. 


\section{Visuals as Projective Techniques in Qualitative Interviews}

Visuals such as drawings, pictures and maps can be usefully employed in qualitative interviews as projective techniques to elicit comments from the participants (Banks, 2007; Greenbaum, 2000; Hoyle et al., 2002; Krueger and Casey, 2000; Stiles, 2004). For example, handdrawings have been used to help respondents articulate deep-seated and often paradoxical emotions about organizational change (Kearney and Hyle 2004; Mazzetti and Blekinsopp 2012; Vince and Broussine 1996; Zuboff 1988). Recently, Slutskaya et al. (2012) used photoelicitation to enable working-class men to engage in reflexive practice by producing more elaborate and expressive accounts of their work experiences.

As explained by Anastas (1994), visuals initiate respondents' imagination and verbal responsiveness: When asked directly - for example about a new product - they might be tense and remain quiet. But if they can express their opinion through pictures or photographs, they answer more promptly and tell richer stories. Through the concealed intent and indirectness of visual projection, respondents can in fact overcome inhibitions and reveal their thoughts and feelings (Steinman, 2009). At the same time, projective techniques reduce the cognitive demands to respondents, by leveraging the intuitiveness of the visual language to transgress communication barriers. Visual stimuli also increase participants' recall and memories of past experiences, by activating an unfolding cascade of visual and verbal associations (Harper, 2002; Wheeldon, 2011; Wheeldon and Faubert, 2009).

Besides assisting the exploration of thoughts and feelings, visuals are widely employed for capturing the cognitive maps of organizational actors, and in turn for exploring multidimensional organizational constructs - such as environmental enactment, industry dynamics, and strategic groups (see also the first project example in this article). In this way, visual data are instrumental to move beyond mechanical models of organizations, towards a vision of organizations as "systems for creating meaning" (Meyer, 1991: 232). Calori et al. (1994) and Huff (1990) have linked cognitive maps to organizational strategy, and explored why similar organizations make sense of, and respond to similar situations in different ways. Meyer (1978) and more recently Crilly et al. (2006) used visual stimuli for gathering data on environmental enactment (Weick, 1979) - i.e., how actors enact and structure the internal and external environment of the organization.

In reviewing the literature, we have identified four distinct approaches to visual projection (i.e., association, completion, expression and collection), based on the type of cognitive stimulation offered by the visual: 


\section{Association}

In the association approach, photographs are used to stimulate the participants' thinking, and to help them articulate their thoughts about the discussion topic. In general, the interviewees are asked to express their impressions about pictures portraying people (personality associations), or situations (situational associations) that are connected to the discussion topic. Alternatively, the participants may be invited to indicate which of several photographs in a stack most closely relate to the subject under discussion (forced associations). The association technique enables the interviewer to uncover deep insights, as the participants tend to 'project' their thoughts of the discussion topic onto the pictures stack (Banks, 2007; Greenbaum, 1998; Krueger and Casey, 2000). For example, Van Riel et al. (1998) has profiled the corporate image and reputation of five airline companies, by asking respondents to draw associations with a set of pictures expressing different kinds of personality traits.

\section{Completion}

Another type of projective technique used in qualitative interviews is picture completion (Greenbaum, 1998). In the cartoon completion test, for example, the participants are presented with an incomplete comic-strip story and are asked to add statements into the empty balloons (Steinman, 2009; Van Dyke, 2009). The picture completion stimuli help the participants to delve more deeply into their minds, and hence to articulate their thoughts with greater fluency and richness of details. A similar stimulation is offered by mind mapping, where the participants are invited to complete a map of thoughts and associations, starting from a central theme suggested by the researcher. As argued by Wheeldon and Ahlberg (2012: 91), mind mapping can be usefully conceived of as a means to "prime the pump" of participant reflection. The completion of mind maps, in particular, was found to unlock unique memories of organizational experiences by enabling respondents to go beyond the rehearsed narratives typical of verbal expression (Wheeldon, 2011).

\section{Expression}

In expressive drawing, participants are asked to provide their reaction to a discussion topic by drawing a picture. At the end of the task, the interviewer asks the respondents to comment on the meaning of their pictures, and to elaborate on the relationship with the discussion topic. For example, Kearney and Hyle (2004) have used expressive drawings to elicit participants' emotional reactions to organizational change, and integrated visual data with verbal interpretations for accuracy and triangulation. In focus groups, the facilitator can involve the entire 
group in developing interpretations of each other's drawings, and hence of the discussion topic (see Bryans and Mavin, 2006). Expressive drawings can be very useful to engage the respondents, and to elicit emotional data that otherwise might not be generated with traditional verbal techniques (Greenbaum, 1998; Stiles, 2004; Krueger and Casey, 2000).

\section{Collection}

In this approach, participants are asked to collect, and to comment on a number of images that relate to the topic being discussed. For example, in the collage technique the interviewees either individually or in group - are given a pile of newspapers, scissors, glue and a blank piece of paper to make a collage expressing their thinking about the topic under discussion (Krueger and Casey, 2000). In the photo-elicitation technique, participants are asked to use photo or video cameras to capture aspects that they consider important or representative of their living or working experiences (Bramming et al. 2012, Slustkaya et al. 2012). At the end of the task, the interviewees are invited to comment on their collection or collage of pictures, and to explain the rationale behind their choice of images. These techniques have been used in a variety of fields, ranging from anthropology to marketing - where they have proven useful to elicit the values, typical users, and purchasing motives associated with new products (Colakoglu and Littlefield, 2011).

The above techniques span across a broad variety of research traditions, being employed by researchers with different epistemological and ontological assumptions as to the role of graphic elicitation. As pointed out by Slutskaya et al. (2012, p. 18), the reading of the term "elicitation" is an object of wide debate in the context of visual methodologies, and a dividing line can be drawn between studies where visual projection is used to elicit pre-existing knowledge from the respondents (e.g., Crilly et al. 2006), and studies where visual projection provides an occasion to co-construct knowledge in the encounter between the researcher and the participants (e.g., Pink, 2001). The first perspective assumes the existence of relatively stable structures of knowledge, and conceives visual projection as a means for the researcher to get deeper access to the thoughts of the interviewees. This perspective is generally theory-driven, and favours the use of visual stimuli that are prepared in advance by the researcher - as in the association approach - to keep a steady focus on the interview areas.

The second perspective, on the other hand, assumes that knowledge is produced and reproduced in the research encounter, and sees visual projection as a collaborative space where the researcher and the informant take part in the construction of meaning (Slutskaya et al. 2012). 
In this perspective, the use of visual stimuli can be conceived as a form of collaboration where research is conducted with people, and not on people (Bryans and Mavin, 2006; Vince and Broussine, 1996). The tendency to use visual projections that are produced by the participants, and refined in conversation with the researcher - as in the expression approach - further enhances the collaborative nature of this research perspective.

In our work we have relied on both research traditions. As a foremost objective, we have used visual projection to encourage the interviewees to recount their real-life experiences, and to elicit the mental constructions that they have formed in the course of repeated social interactions. At the same time, we acknowledge that meaning is framed and reframed in the research encounter, and we accordingly engage in a collaborative interpretation of the meaning assigned to the visual stimuli. In so doing, we have realized that visual projection does contribute to building rapport between the researcher and the participants, especially by providing greater emotional engagement than purely verbal accounts.

Nevertheless, we have also become aware of the limitations of visual projection, recognizing that - besides rapport building - most communication challenges inherent in qualitative interviews were not adequately met through the visual stimuli. Visual projection has not greatly helped to overcome knowledge barriers between the interviewer and the interviewee, or to build a shared language between different communities of practice involved in a focus group. If the interviewees do not fully understand the perspective of the interviewer, or lack the literacy to express themselves through the visual language, they may provide vague or tentative answers to the visual stimulation. In other cases, the visual stimuli - especially if prepared in advance by the researcher - may provide a too narrow frame, therefore occluding, rather than exposing the interviewees' knowledge (Umoquit et al. 2008).

A further disadvantage of projective techniques lies in the complexity of the analysis of data: In order to avoid misinterpretations, visual data should be integrated with verbal interpretations by the participants (Greenbaum, 1998) - yet, some respondents may feel uncomfortable with projective exercises (Steinman, 2009) or may fail to articulate their thinking while producing their visual representations. In the latter case, the researcher lacks full understanding of the image-in-use, and may be led astray by the temptation to take the visual stimuli itself as the site of analysis.

In the next section, we thus explain how a shift from visuals as projective techniques towards visuals as facilitation techniques may enhance qualitative interviewing by enabling the researchers to more fully exploit the potential of the visual language. To make the case for visual facilitation in qualitative interviews, we draw on the literature on knowledge visualization, 
which suggests that real-time visualization of group conversations fosters the co-construction of knowledge. From the literature on organizational knowledge, we further take the suggestion that visuals can act as 'boundary objects', therefore facilitating the translation of knowledge across communities of practice. As the next section will make clear, visual facilitation pushes forward the agenda of building a collaborative rapport with the participants, while also strengthening the researchers' focus on the process - not just the end product - of visual interviewing.

\section{Visuals as Facilitation Techniques in Qualitative Interviewing}

Although being often used to moderate business meetings (Buzan and Buzan, 2006; Kuchenmüller and Stifel, 2005; Sibbet, 2010), visual facilitation has never been employed systematically for research purposes (i.e., interview data collection and analysis). Nevertheless, a large body of literature suggests that visual facilitation is likely to increase the quality of knowledge sharing in face-to-face conversations (Eppler and Burkhard, 2007; Isenberg et al. 2011). The collaborative use of visual representations has been found to reduce the challenges inherent in knowledge-intensive conversations (Mengis and Eppler, 2006), to foster the elicitation of experiential knowledge (Bresciani and Eppler, 2009), and to increase mutual orientation among participants (Comi and Eppler, 2011). Such effects have been shown in a variety of application domains, ranging from strategic management (Eppler and Platts, 2009), to project work (Whyte et al., 2008), all the way to learning and education (Fischer et al., 2002).

Building on these findings, we suggest using visual facilitation in field research and propose an approach where visuals are drawn simultaneously by the researcher and the participants during interviews. The visual language is thus integrated into the research process and shifts from being an external stimulus (to prompt answers) to becoming an integral component of the research method. As an example, the facilitator in focus groups may use visualization tools such as visual templates, sticky notes, and e-moderation software to lead a discussion, and at the same time enable participants to write and/or draw out their thoughts. In particular, visual templates - printed on a poster, or loaded on software - provide a canvas where participants can document their discussion with mapping techniques. As suggested by Kuchenmüller and Stifel (2005: 394), by filling visual templates the individual or the group "answers important questions while respecting definite rules".

In comparison to purely text-based facilitation like flipchart handwriting, visual facilitation provides a more engaging experience for participants (Buzan and Buzan, 2006), and in turn 
will foster an active involvement in the interview process. Moreover, visual representations play a crucial role in the sense-making of individuals and groups, by bringing the advantage of representational guidance (Suthers, 2001: 257). In fact, visual representations provide an overarching structure which organizes information, coordinates the conversation, and highlights key aspects. Furthermore, the empty fields in a visual template will maintain interaction throughout the interview process. They provide an opportunity for the interviewees to fill in their contributions and to engage in a discussion of the contents being mapped.

By enabling greater visibility of discussion threads, visual facilitation can draw attention to the interview themes and help both the researcher and the interviewee to keep track of the conversation. In focus groups, this will lead to the creation of a group memory (Kuchenmüller and Stifel, 2005), which reduces the participants' difficulties in following the conversation, and building on each other's contributions. This advantage might extend also to online focus groups, where visual facilitation is likely to increase the fitness of participants' contributions, by providing tangible orientation and signposts during the conversation.

Facilitation techniques may also reduce knowledge asymmetries between the researcher and the researched, since the co-construction of visual artefacts provides a mechanism for building shared understanding across knowledge boundaries (Carlile, 2002). In this regard, visual representations act as 'boundary objects' - i.e., objects that are robust enough to maintain a common identity across sites, yet plastic enough to adapt to the local needs of the different parties (Star and Griesemer, 1989). The template background conveys the knowledge expectations of the interviewer, and at the same time adapts with the knowledge expressions of the interviewee. Furthermore, the visual language provides common conventions for translating meaning, and clarifying differences between communities of practice - such as academia and industry.

Visual facilitation has been shown to bring about other group interaction advantages, namely facilitating balanced participation of group members and reducing the potential for interpersonal conflict (Mengis and Eppler, 2006). When the conversation is mediated by visuals, participants are not pointing directly at each other, but rather express their agreement or disagreement towards impersonal objects, such as notes on a visual template (Comi and Eppler, 2011). In a study of business meetings, Kuchenmüller and Stifel (2005: 388) consistently noticed that "it is easier to offend and attack using words than images". Therefore, the depersonalization effect of visuals is particularly beneficial in focus groups to reduce social pressure and encourage participants to freely express their dissent with the rest of the group. 
Furthermore, the problem of 'groupthink' or excessive harmony seeking in focus groups might be reduced by having participants first write their contributions individually before the facilitator maps or visualizes them in the plenary session on a joint poster or projected space. In this way, the contributions of shy participants are collected and emphasized as well as integrated into the conversation process. Groupthink in such settings can be further reduced by changing the way that questions are asked (for example only visually and not verbally) and by encouraging participants to play devil's advocate and question their own contributions (see Macgougall and Baum, 1997). In visual focus groups, this can be done discreetly and without exposing oneself, for example by flagging elements visually using icon cards or sticky notes.

At the end of the interview, showing the filled template to the participants offers the possibility to gather further data, by asking probing questions, delving into important aspects, and commenting on the main findings. Therefore, the visual used for facilitation is re-injected into the interview process, and used as a stimulus for the elicitation of additional insights, in a virtuous cycle between facilitation and projection. As regards the final stage of data analysis, the use of templates will facilitate the aggregation and the comparison of data collected across multiple interviews or focus groups. In the next section, we present two projects from our research where we have used visuals for projection and facilitation, and we subsequently elaborate on the benefits of integrating such techniques in a research endeavour.

\section{Examples from the Field [2]}

In two research projects, we have conducted one-to-one interviews and focus groups, making use of visualizations for projective as well as facilitation purposes. We have used sketches to illustrate the interview process (see Figure 1) and templates populated with the participants' comments (see Figures 2 and 5). At a later stage, we have compared our experiences by engaging in research conversations, and we have further elaborated on the synergies between visual projection and facilitation. In turn, this 'reflective practice' laid the foundations for the development of an integrative approach to visual interviewing. Before introducing this approach, we provide a few examples of the methodological insights derived from our research experience.

\section{First research experience: Knowledge communication in a community of practice}

In a first research project, we have conducted 28 one-to-one qualitative interviews for the purpose of gathering expert information on the difficulties and solutions for communicating 
knowledge among natural hazard and risk managers. Subsequently, we have analysed the data and presented the results to a group of respondents from the same community of practice, in the course of two focus group discussions of 8-10 participants each. By so doing, we have aimed to validate preliminary findings and to extend the interview results with further insights from the professional community.

The interview participants were selected following a purposive sampling strategy (Spencer et al., 2003); more precisely, we have chosen a homogeneous sample to acquire an in-depth understanding of habits, routines and thinking in this professional community. In the course of each interview (90-120 minutes), the respondents were asked to provide information about their professional experience and their knowledge of natural hazards management. In particular, they were invited to explain how they acquired and shared their professional knowledge using artefacts such as risk maps, online-planning tools or strategy reports. A further objective of the interview consisted of mapping the boundaries of the community of practice, by exploring mutual relationships within a constellation of actors.

Challenges at the very beginning of the interviews, such as establishing rapport, gaining trust, understanding and speaking the language of respondents, were reduced by using an association stimulus, i.e. a sketch of the interview process (Figure 1). This sketch was employed not only as an 'ice-breaker' at the beginning of the face-to-face interview, but also as a reference to structure the interview content, and to ensure coverage of all the relevant discussion topics. This overview sketch was also used as a facilitation technique during the interview process: It was positioned on the interview table and functioned as a reference whenever a new interview topic was addressed. In this way, the interviewer was able to refer to the sketch and show the progress of the interview. In turn, this helped the interviewee follow the sequence of the interview and refer back to things already said.

Insert Figure 1 about here

During the interview we also introduced a visual timeline, loaded on a facilitation software (Figure 2). This interactive template was used to explore the interviewees' awareness and knowledge of important developments in their professional community (such as the introduction of online-planning tools). The respondents were asked to fill out the empty template by reflecting on the evolution of their profession, taking into consideration natural hazards events, laws and directives, and processes of knowledge transfer within the community. This 
completion stimulus addressed the challenge of gathering high-quality data by literally bringing implicit knowledge, experiences and opinions to the table.

Insert Figure 2 about here

The participants indicated that they liked the template completion task, and provided unsolicited positive feedback. Often, there was a moment of surprise in the beginning, which then turned into 'fun' and a desire to fully complete the task. While filling out the template, the interviewees kept on talking in a 'thinking aloud' mode - a phenomenon which is reminiscent of certain forms of ethnographic interviewing (Fontana and Frey, 1994). Furthermore, the template worked as a 'boundary object' (Star and Griesemer, 1989), and spanned knowledge boundaries between the interviewer and the participant by providing multi-dimensional communication (verbal and visual) and joint focus. As a result, the collaborative work around the template brought up experiences, opinions and expert knowledge which probably would not have been shared in a purely verbal setting.

Besides contributing to a shared understanding, the use of visual templates yielded practical advantages as well, for example freeing the researcher from the need of taking extensive notes of the interview content. As the participants were recording their thoughts on the visual template on their own, the interviewer had more time to concentrate on non-verbal aspects of the interview (such as body language). As researchers, we were therefore able to observe the process of working with visual templates from a meta-perspective, and we could take side notes of the interview process. These side notes - concerning for example the interviewees' reactions to using visual templates - helped to refine our interviewing skills, and to inform the development of our integrative approach to visual interviewing. Therefore, this occasion of reflexivity - afforded by the use of visual templates - provided an additional source of data generation and collection.

Following the visual timeline, we used a card sorting exercise. In this task we invited the interviewee to organize and to comment on cards representing different professional roles, experts and organizations involved in natural hazards management (Figure 3). This enabled us to better understand the configuration of 'players' within this community of practice. The analysis of the sorted cards involved a software program and resulted in a dendrogram (Figure 4) describing the resulting groups of actors (with percentages indicating the interviewees' agreement about actors' inclusion in a given group). 
Insert Figures 3 and 4 about here

A few weeks after these one-to-one interviews, we invited some of the same, plus new participants to join a focus group in order to discuss the preliminary interview results. As mentioned above, we conducted two focus groups of 8-10 participants each, for an average duration of 1,5 hours. We showed the dendrogram in Figure 4 to the focus group participants as a stimulus for discussion and as a device for new data collection. As suggested above, one of our primary objectives for the interviews and focus group discussions consisted of mapping the boundaries of the community of practice. Without the dendrogram, the actors' constellation would have been very difficult to describe to the focus group participants. By using this visualization, it became clear which groups existed and which actors seemed to be outsiders. The ensuing discussion in the focus group was very rich, came straight to the point (how do you perceive these groups of community players?) and the participants were able to 'attach' their opinions to the visualization. While the discussion was going on, the facilitator took notes on the computer and simultaneously showed the emerging picture on the wall. Thus, every participant was able to follow the discussion and immediately adjust his or her input if necessary. Through this procedure we ensured that ideas and opinions were collaboratively elaborated and evaluated by multiple experts in the same disciplinary area. This procedure enabled another kind of reflexivity: The focus group participants not only collaborated with the researcher, they also initiated a discussion around the visualization of previous results, thus collectively clarifying their prior individual view points and validating them jointly.

\section{Second research experience: Knowledge sharing in inter-organizational settings}

In a second research project, we conducted four focus groups of 8-10 participants each to evaluate the use of a software application for supporting knowledge sharing and development in inter-organizational settings. Regarding the research design, we adopted a mixed methods approach, with the focus groups being conducted to follow up an experiment. In the experiment, we compared the performance of software-supported and control groups and this by assessing the number of information elements shared, and the quality of solutions developed. We introduced the focus group study to clarify unexpected experiment findings and to gather deeper data on the research topic (see Morgan, 1996 and Morgan, 1997, on the use of focus 
groups in combination with other research methods). Out of the 229 experimental subjects, 35 were randomly selected to serve as focus group participants. In the course of the focus group - which lasted about 45-50 minutes - participants were asked to reflect on their experience in the experiment and to provide real-life accounts of software use in inter-organizational settings.

Insert Figure 5 about here

To conduct the focus group, we used a visualization software for group facilitation (let's focus, see: en.lets-focus.com) connected to a video projector, so that participants could simultaneously view the facilitator's screen. For each interview question, the facilitator populated a visual template (loaded as a digital file on let's focus) with contributions from the participants (see Figure 5 for a snapshot of a completed template taken after a focus group discussion). In this regard, the visualization software was used for real-time documentation, moderation, and summarisation of the focus group discussion. The visual templates provided a "collective memory", enabling participants to keep track of the discussion and to build on each other's contributions with greater ease.

As compared to previous studies - in which we moderated focus groups without visual facilitation - we noticed a tendency to follow a more disciplined turn-taking in the conversations. In effect, visual templates provided signposts throughout the conversation, therefore introducing implicit rules for turn-taking and reducing the risk of dominance on the part of "much talkers". Without assuming a central role in the team conversation, the facilitator was able to prompt contributions from all the participants, mostly by taking advantage of the circular distribution of participants around the projected template. The physical setting was therefore conducive to a balanced discussion around the visual template and created a relaxed atmosphere where participants could comfortably talk together.

Most importantly, the visual templates worked as 'boundary objects' and provided interpretive flexibility: as such, they allowed participants to assign local interpretations, while at the same time building shared understanding (Star, 2010). In this follow-up study, in fact, different groups were able to work together without deep knowledge of each other's experience in the previous experimental sessions. As the interaction unfolded, the visual template became the focus of participants' communication, and a repository of the knowledge created through group interaction. 
As they provided a synopsis of the group interview areas, the visual templates represented an important resource in the analysis of data, and were therefore used as a framework for content-coding the interview transcripts. In analysing the data, we developed a summary visualization displaying common - as well as unique - themes that emerged from the four focus group sessions. As a procedure for checking the accuracy of data analysis, we brought the main findings of each focus group back to the participants, and asked them to provide feedback. The analysis of the focus group discussion was then integrated with the results of the experimental session, and delivered to the participants in the form of a plenary presentation. To a large extent, the focus group participants confirmed the findings of the data analysis, while also offering further information and clarifications.

Being aware of the novelty of adopting visual facilitation for research purposes, we engaged in research debriefing conversations to compare, and derived lessons learned from our experiences in conducting visual focus groups (see also the Discussion section for this point). Such a reflexive practice led to a progressive refinement of our interviewing steps, and contributed to the development of the integrative framework presented in the next section.

\section{Integrating Visual Projection and Facilitation}

From the researcher's perspective, the interviewing process can be viewed as comprising four stages: First the introduction of the topics to be discussed, then the facilitation of the interview itself, then the wrap-up of the discussion, and finally the analysis of the generated data (Fontana and Frey, 1994; Hubermann and Miles 1994). Each phase has its own challenges, many of which can be overcome using visualizations - most notably by integrating projective and facilitation techniques. Based on our own experience, we suggest using visualization throughout all interview phases - first for clarifying the interview scope, then to stimulate answers from the respondents and later on to facilitate the interview process by means of graphic summarization, clarification or itemization. More specifically, here are the advantages of visuals for each stage:

\section{Initiating the Interview}

Challenges at the very beginning of qualitative interviews, such as establishing rapport, clarifying the interview scope and purpose, gaining trust, understanding and speaking the language of respondents can be overcome by using an association stimulus, such as a sketch of the interview process. Such an informal sketch can show how the different topics to be discussed 
inter-relate. It can give the interview participants the certainty of knowing what's coming and what is expected of them.

\section{Conducting the Interview}

During the interview, the researcher is faced with the difficulty of keeping track of what has been said and at the same time leading the interview. Probing on what the interviewee has said before - while conducting the interview - may be difficult in a purely verbal setting. Here, visual templates help to structure, complete, and document what has been stated. In focus groups, they provide a collective memory of the discussion, and are instrumental to encourage participants to build on each other's contribution. Additionally, templates can promote so called 'thinking aloud', i.e. motivate the subject to talk about things and experiences, which probably would not be mentioned without the visual support. The interviewer can take side notes while the subject is thinking aloud. Often, these verbalised thoughts are valuable information and can be used in addition to the analysis of transcripts and completed templates.

\section{Wrapping-up the Interview}

At the end of the interview, the researcher can summarize the main points that have emerged in the interview process by showing the completed template to the interviewee(s). By wrapping up an interview with visual representations, the interviewer can stimulate respondents to further delve into relevant topics or to clarify misunderstandings. Visual templates that have been completed collaboratively during the interview process create trust and leave the interviewee with the feeling that his or her input has been acknowledged, recorded and will be properly analysed.

\section{Analysing data}

Depending on the research questions, the researcher can adopt different approaches to the analysis and interpretation of visual data (see also Miles and Huberman, 1994; Ryan and Bernard, 2003). In our experience, the integration of verbal (e.g., interview transcript) and visual data (e.g., filled templates) is paramount to shed light on aspects that may elude the researcher during the interview process. The interview transcript can therefore be analysed in parallel with the visual produced in the research encounter, reviewing both from a meta-perspective. When there are video recordings in addition to the filled templates, analysing the process of template filling - i.e., how participants arrange, move and change textual notations onto the template background - will add greater richness to the data (visual focus group software such as lets-focus.com record the on-screen movements automatically, so that the process of tem- 
plate filling can be "replayed" by the researcher later on). If necessary, the researcher can explore individual or group differences by comparing the visual templates filled out by different categories of interviewees and participants. Furthermore, a summary visualization can be produced by integrating common and unique themes that emerged from the interview transcripts and the completed templates. Such a summary visualization is useful to present findings and can be re-injected in the research process, for example by inviting participants to join a follow-up meeting. In our experience, this process is useful to validate findings. It provides the occasion to check the researcher's interpretation against the participants' explanations, and to explore important themes in more detail.

In the following table, we have summarized this approach and point out the distinctive and complementary advantages brought about by visual projection and visual facilitation.

Insert Table 1 about here

This practical approach is offered to provide researchers with general guidance in using visual projection and facilitation for qualitative interviewing. It is not intended as a set of rigid rules. Other researchers should flexibly adjust this approach to their relationship with the interviewee(s), their research settings and contexts, as well as their research domain and topic. While refraining from a 'one-size fits all' approach, we do suggest that visual interviewing should be informed by the underlying principle of exploiting the visual language throughout the entire research process. At the same time, the participants should be actively involved in the construction, discussion, and interpretation of visuals - in line with a vision of qualitative interviewing as a cooperative endeavour.

This integrative approach is grounded in the extant literature on visual methods in qualitative research, and has been developed by the authors reflecting on their experience in conducting one-to-one interviews and focus groups. In turn, practice in qualitative interviewing led to a progressive refinement of the method, and spurred reflexive conversations among the researchers. As will be discussed in the next section, this reflexive practice has resulted in higher awareness of the limits of our approach, and highlighted the need to consciously switch between visual and verbal modes of interviewing. 


\section{Discussion}

Based on our experience, we have observed several advantages of integrating visual projective and facilitation techniques in one-to-one and focus group interviews. In the early stages of the interview, projective techniques brought about communicative advantages such as building rapport, and eliciting deeper answers by removing the respondent's inhibitions. During the interview process, visual facilitation provided further communicative advantages, by fostering articulation, integration, and co-construction of knowledge. As boundary objects, the visual templates were flexible enough to accommodate the needs of the interviewer and interviewee(s), and robust enough to supply common meaning across sites. By providing a common reference during the interview, the visual templates enabled both parties to build on each other's contributions, and to keep track of the discussion as it unfolded over time. In focus groups, the communicative advantages of visual facilitation extended to spanning knowledge boundaries among multiple respondents. While it is undeniable that focus group members affect each other in their answers (Macgougall and Baum, 1997: Morgan, 1996; Sussman et al., 1991) we have suggested that visual facilitation produces a "depersonalization effect" which may contribute to reduce biases related to group interaction (e.g., conformity pressure and groupthink). Future research should explore this aspect in greater detail, with the aim of gathering empirical evidence on the impact of visual facilitation on group dynamics occurring in focus groups.

Finally, visual facilitation provided analytical advantages for the subsequent phases of our qualitative research projects: By using visual templates for data collection and analysis, we have increased the possibilities of comparing data collected across multiple interviews. By doing so, we were able to identify contrasts and comparisons, which is a classic tactic to improve our understanding and sharpen our findings (Huberman and Miles, 1994). Moreover, the aggregated data were presented in a visual format to participants and/or other members of the informant community for validation purposes, and hence re-injected in the interview process as visual stimuli to promote further discussion and acquire new data. On occasion, the use of visual templates during the facilitation process has enabled us to note side information mentioned incidentally by the interviewee, and to elicit nuances that led to unexpected findings.

While providing considerable advantages, the use of visual facilitation for qualitative interviewing is not without limitations: The effectiveness of the interview is highly dependent on the visual literacy of the interviewer or moderator and his or her ability to use visual facilitation tools and software swiftly. Before the qualitative interview, the researcher should careful- 
ly choose visual templates based on the topic under investigation. For the selection of visual templates, the extant literature on knowledge visualization may provide a useful guidelines (Eppler and Burkhard, 2007).

As another potential limitation or risk of visual interviewing, respondents may be 'locked' into the visual templates and neglect discussing relevant issues that are not emphasized by the visual structure. This is the negative side of the "representational guidance" provided by visual representations (Suthers and Hundhausen 2003: 186). The visual structure in fact provides guidance but at the same time "constrains which knowledge can be expressed in the shared context, and makes some of that knowledge more salient and hence a likely topic of discussion". When asked to reflect on their experience with visual facilitation, participants consistently recognized that visual templates provided a structure to organize the discussion. Nevertheless, a few participants mentioned that they felt somehow constrained by such a structure, since it did not afford unstructured thinking and free flow of associations. Furthermore, we are aware of the "cajoling effect" of visual representations (Bresciani and Eppler, 2009), which may cause respondents to develop overly positive attitudes towards the interview or discussion topic.

In order to mitigate the biasing effects of visual representations, the interviewer may find it useful to temporarily put the visual aside and probe the respondents' answers in a purely verbal mode. To reduce the negative effects of representational guidance, the interviewer should motivate the respondents to think beyond the categories prompted by the visual template and hence to engage in a free-flow of thoughts. The cajoling effect can be corrected by asking the interviewees to critically revise their statements, and to substantiate their viewpoints with further arguments or evidence. In turn, this requires an ability to promptly detect biased answers, and to masterfully intertwine visual and verbal modes of questioning.

Moreover, the simultaneous conduction and visualization of the interview poses many challenges for the researcher: While it is advisable to have a facilitator and an interviewer working together, the coordination among the two researchers may be difficult or even bias the interview process. Finally, there is always the possibility that some participants may feel uncomfortable seeing their contributions visualized and thus explicitly recorded (especially when they mention sensitive issues). In such cases, the act of making the participants' contributions visual may make them less forthcoming and outspoken (although we have not witnessed such behaviour in our research projects thus far).

As mentioned repeatedly throughout the article, our practice with visual methods has prompted several occasions for reflection, which have informed the development of our integrative 
approach to visual interviewing. The use of facilitation techniques and the introduction of visualization software provided room for greater reflexivity by enabling us to concentrate on non-verbal, and often neglected, aspects of the interview. Furthermore, we deliberately engaged in reflexive practice, by initiating debriefing discussions about our research experiences and making an attempt to derive lessons learned. Not only were we able to retrospectively appreciate the advantages and the limits of using visual methods, but we also became aware of the importance of switching between verbal and visual modes of interviewing. Furthermore, we were able to further reflect about our expectations as researchers and to acquire greater awareness of our epistemological position. While confirming our epistemological standpoint as one of moderate constructivism, we have reached a higher level of understanding as to the dynamics underlying the construction of reality in the interview encounter. Most importantly, we have realized that visual techniques are not neutral instruments in the hand of researchers, but rather contribute actively to shape data generation. In this respect, visual representations can be conceived of as non-human agents that provide guidance, but also constrain, or cajole the respondents throughout the interview process. The skilful researcher should therefore use them judiciously, in way consistent with the overall research design.

\section{Conclusion}

As the most widely used research technique in management studies, interviews should not be looked at as a research method that is cast in stone. It should rather be seen as a continuously evolving tool that can and should be further refined and adjusted. In the Editorial of the first issue of Qualitative Research in Organizations and Management, Cassell and Symon (2006) have acknowledged the need to provide a showcase for the diverse range of qualitative techniques in use, and at the same time to expand the breadth of qualitative methodologies. This is especially important in organization and management studies, where qualitative research is often evaluated with reference to positivist criteria, and novel techniques still have a long way to go before gaining credibility. To this end, Bluhm et al. (2011) call for management scholars to facilitate the mainstreaming of novel methodologies, by adapting the best practices developed in adjacent fields, such as sociology, psychology, and linguistics.

In this contribution we suggest a methodological development of qualitative interviewing by integrating visual projective and facilitation techniques into the interviewing process. While projective techniques have been used for a long time, we argue that the inclusion of facilitation techniques is instrumental to overcome many challenges inherent in one-to-one and focus 
group interviews. While visual projection is useful to initiate an interview by tapping into the respondents' innermost thoughts, visual facilitation enhances knowledge articulation, coconstruction, and integration during the interview. We have proposed a rationale for projective and facilitation techniques based on a review of literature and our own research and we have accordingly pointed out the advantages and limitations of such visual techniques.

From a theoretical perspective, we also contribute to advancing the collaborative perspective on visual methodology by arguing for a greater involvement of informants in shaping the research encounter. While projective techniques can be effectively used to foster collaboration between the researcher and informant (Bryans and Mavin, 2006; Harper, 2002; Slutskaya et al. 2012; Warren 2009; Vince and Broussine, 1996), the introduction of visual facilitation takes this research perspective a step forward. In projective techniques, the informant is presented with visual stimuli predefined by the researcher (association, completion), or is required to freely express his or her thoughts with visual techniques (collection, expression). In facilitation techniques, the interviewer and the interviewee are reciprocally engaged in the coconstruction of the visual material, and collaboratively shape the conduit of the interview process. We have further suggested that the visual material - by virtue of its interpretive flexibility - can function as a boundary object transgressing the knowledge barriers between researcher and informant. By integrating projective and facilitation techniques, the involvement of the interviewee is extended to all stages of the interview process - from the initial stage where the visual stimuli is being presented, to the final stage where the interview is wrappedup with visual templates that had been collaboratively filled out throughout the interview process.

As we experienced during our own research work, it seems to be important that the researcher is not fixed or hooked on the visual at any stage of the interview, but uses the visual as an option. Management of one's own expectations as a researcher seems to be important as well. We therefore suggest understanding facilitation and projection techniques as process catalysts and as devices to trigger conversations. Using visualizations during interviews may also lead to more comprehensive data by triggering more reflexivity: while the interviewee is sketching or drawing, the interviewer may take notes on this process and can use this information in addition to the transcripts for subsequent interpretation.

In spite of this promising potential, the strengths of visualization methods in qualitative interviews need to be substantiated with further application examples and empirical evidence in order to build solid methodological foundations. In future studies, researchers should evaluate the added value of visual facilitation in qualitative interviewing by comparing the richness of 
data gathered in visualization-based interviews with that from purely verbal interviews. Also, our practical approach for using visualizations in one-to-one interviews and focus groups has to be further developed, tested, evaluated and discussed in academia. Still, we hope to have shown that the sense making mantra first evoked by Karl Weick (1979) is indeed highly relevant - taken literally - for the interview process: How can we know what they think until we see what they say? 


\section{References}

Alvesson, M., and Sköldberg, K. (2009), Reflexive Methodology: New Vistas for Qualitative Research, SAGE, London.

Anastas, M., (1994), "Visuals stimulate richer response in focus groups and individuals interviews." Quirk's Marketing Research Review.

Banks, M. (2007), Using Visual Data in Qualitative Research, SAGE, Thousand Oaks, CA.

Bluhm, D. J., Harman, W., Lee, T. W. and Mitchell, T. R. (2011), "Qualitative Research in Management: A Decade of Progress." Journal of Management Studies, Vol. 48, No. 8, pp. 1866-1891.

Bramming, P., Hansen, B. G., Bojesen, A., and Olesen, K. G. (2012). (Im)perfect pictures: snaplogs in performativity research. Qualitative Research in Organizations and Management, Vol. 7, No. 1, pp. 54-71.

Bresciani, S. and Eppler, M. J. (2009), "The Risks of Visualization: a Classification of Disadvantages Associated with Graphic Representations of Information.", in Schulz, P. J., Hartung U., and Keller, S. (Eds.) Identität und Vielfalt der Kommunikationswissenschaft, UVK Verlagsgesellschaft mbH, Konstanz (Germany).

Bryans, P. and Mavin, S. (2006), "Visual images: a technique to surface conceptions of research and researchers." Qualitative Research in Organizations and Management, Vol. 1, No. 2, pp. 113-113.

Bryman, A. and Cassell, C. (2006), "The researcher interview: A reflexive perspective." Qualitative Research in Organizations and Management, Vol. 1, No. 1, pp. 41-55.

Buzan, T. and Buzan, B. (2006), The Mind Map Book, BBC Active, Essex, UK.

Calori, R., Johnson G. and Sarnin, P. (1994), "CEOs' cognitive maps and the scope of the organization." Strategic Management Journal, Vol. 15, No. 6, pp. 437-457.

Carlile, P. R., (2002), "A Pragmatic View of Knowledge and Boundaries: Boundary Objects in New Product Development." Organization Science, Vol. 13, No. 4, pp. 442-455.

Cassell, C. and Symon, G. (2006), "Taking qualitative methods in organization and management research seriously." Qualitative Research in Organizations and Management, Vol. 1, No. 1, pp. 4-12.

Colakoglu, S. and Littlefield, J. (2011), "Teaching Organizational Culture Using a Projective Technique: Collage Construction." Journal of Management Education, Vol. 35, No. 4, pp. 564-585. 
Comi, A. and Eppler, M. J. (2011), "Assessing the Impact of Visual Facilitation on InterOrganizational Collaboration: an Experimental Study." Journal of Universal Computer Science, Vol. 17, No. 10, pp. 1430-1454.

Crilly, N., Blackwell A. F. and Clarkson, P. J. (2006), "Graphic elicitation: using research diagrams as interview stimuli." Qualitative Research, Vol. 6, No. 3, pp. 341-366.

Davison, J., McLean C. and Warren, S. (2012), "Exploring the visual in organizations and management." Qualitative Research in Organizations and Management, Vol. 7, No. 1, pp. 5-15.

Eppler, M. J. and Platts, K. W. (2009), "Visual Strategizing: The Systematic Use of Visualization in the Strategic-Planning Process." Long Range Planning, No. 2, pp. 42-74.

Eppler, M. J. and Burkhard, R. A. (2007), "Visual representations in knowledge management: framework and cases." Journal of Knowledge Management, Vol. 11, No. 4, pp. 112 122.

Fischer, F., Bruhn, J., Gräsel, C. and Mandl, H. (2002), "Fostering collaborative knowledge construction with visualization tools." Learning and Instruction, Vol. 12, No. 2, pp. 213-232.

Fontana, A. and Frey, J. (1994), "Interviewing. The art of science.", in Denzin N. K. and Lincoln, Y. S. (Eds.) Handbook of Qualitative Research, SAGE, Thousand Oaks, CA.

Greenbaum, T. L., (1998), The Handbook for Focus Group Research, SAGE, Thousand Oaks, CA.

Greenbaum, T. L., (2000), Moderating Focus Groups: A Practical Guide for Group Facilitation, SAGE, London.

Guba, E. G., and Lincoln, Y. S. (1994). "Competing Paradigms in Qualitative research". In N. K. Denzin and Y. S. Lincoln (Eds.), Handbook of Qualitative Research (pp. 105-117). London: Sage.

Harper, D. (2002). "Talking about pictures: a case for photo elicitation." Visual Studies, Vol. 17, No. 1, pp. 13-26.

Hoyle, H. R., Harris M. J. and Judd, C. M. (2002), Research Methods in Social Relations, Wadsworth, US.

Hubermann, M. and Miles, M. (1994), "Data Management and Analysis Methods." In N. K. Denzin and Y. S. Lincoln (Eds.), Handbook of qualitative Research (pp. 428-444). SAGE Publications, Inc, Thousand Oaks, CA US.

Huff, A. S. (1990), Mapping strategic thought, Wiley. 
Huff, A.S. and Jenkins, M. (2002), Mapping Strategic Knowledge, SAGE, Thousand Oaks, CA.

Isenberg, P., Elmqvist, N., Scholtz, J., Cernea, D., Kwan-Liu M., and Hagen, H. (2011), "Collaborative visualization: Definition, challenges, and research agenda." Information Visualization, Vol. 10, No. 4, pp. 310-326.

Kearney, K. S. and Hyle, A. E. (2004), "Drawing out emotions: the use of participantproduced drawings in qualitative enquiry." Qualitative Research, Vol. 4, No. 3, pp. 361-382.

Krueger, R. A. and Casey, M. A. (2000), Focus Groups: A Practical Guide for Applied Research, SAGE, London.

Kuchenmüller, R. and Stifel, M. (2005), "Quality without a name.", in Schuman, S. (Ed.) The IAF Handbook of Group Facilitation, Jossey-Bass, San Francisco, CA.

Macgougall, C. and Baum, F. (1997), "Discussion in Focus Groups The Devil's Advocate: A Strategy to Avoid Groupthink and Stimulate Discussion in Focus Groups." Qualitative Health Research, Vol. 7, 532-541.

Mazzetti, A., and Blenkinsopp, J. (2012). "Evaluating a visual timeline methodology for appraisal and coping research." Journal of Occupational and Organizational Psychology, Vol. 85, No. 4, pp. 649-665.

Mengis, J. and Eppler, M. J. (2006), "Seeing versus Arguing: The Moderating Role of Collaborative Visualization in Team Knowledge Integration." Journal of Universal Knowledge Management, Vol. 1, No. 3, pp. 151-162.

Meyer, A., (1978), "Management and Strategy." eds. R. E. Miles and C. C. Snow, Organizational Strategy, Structure, and Process, McGraw-Hill, New York.

Meyer, A. D., (1991), "Visual Data in Organizational Research." Organization Science, Vol. 2, No. 2, pp. 218-236.

Morgan, D. L. (1996), "Focus Groups." Annual Review of Sociology, Vol. 22, pp. 129-152.

Morgan, D. L., (1997), Focus Groups as Qualitative Research, SAGE, Thousand Oaks, CA.

Pink, S. (2001). Doing Visual Ethnography: Images, Media and Representation in Research, Sage, London.

Reason, P. (1988), Human Inquiry in Action, Sage, London.

Ryan, G. W., and Bernard, H. R. (2003). Techniques to Identify Themes. Field Methods, Vol. 15, No. 1, pp. 85-109.

Sibbet, D. (2010), Visual Meetings: How Graphics, Sticky Notes and Idea Mapping Can Transform Group Productivity, John Wiley \& Sons, Hoboken, New Jersey. 
Slutskaya, N., Simpson, A., and Hughes, J. (2012). "Lessons from Photoelicitation: Encouraging Working Men to Speak". Qualitative Research in Organizations and Management, Vol. 7, No. 1, pp. 16-33.

Spencer, L., Ritchie, J., Lewis J. and Dillon, L. (2003), "Quality in Qualitative Evaluation: A framework for assessing research evidence ", National Centre for Social Research, London.

Star, S. L. (2010), "This is Not a Boundary Object: Reflections on the Origin of a Concept." Science Technology Human Values, Vol. 35, pp. 601-617.

Star, S. L. and Griesemer, J. R. (1989), "Institutional Ecology, 'Translations' and Boundary Objects: Amateurs and Professionals in Berkeley's Museum of Vertebrate Zoology, 1907-39." Social Studies of Science, Vol. 19, No. 3, pp. 387-420.

Steinman, R. B. (2009), "Projective Techniques in Consumer Research." International Bulletin of Business Administration, Vol. 5, pp. 37-45.

Stiles, D. R., (2004), "Pictorial representation.", in Cassell, C. and Symon, G. (Eds.) Essential guide to qualitative methods in organizational research, Sage Publications.

Sussman, S., Burton, D., Dent, C. W., Stacy A. W. and Flay, B. R. (1991), "Use of focus groups in developing an adolescent tobacco use cessation program: collective norm effects." Journal of Applied Social Psychology, Vol. 21, No. 21, pp. 1772-1782.

Suthers, D. D., (2001), "Toward a Systematic Study of Representational Guidance for Collaborative Learning Discourse." Journal of Universal Computer Science, Vol. 7, No. 3, pp. 254-277.

Suthers, D. D., and Hundhausen, C. D. (2003). An Experimental Study of the Effects of Representational Guidance on Collaborative Learning Processes. Journal of the Learning Sciences, Vol. 12, No. 2, pp. 183-218.

Umoquit, M., Dobrow, M., Lemieux-Charles, L., Ritvo, P., Urbach, D., and Wodchis, W. (2008). The efficiency and effectiveness of utilizing diagrams in interviews: an assessment of participatory diagramming and graphic elicitation. BMC Medical Research Methodology, 8(1), 53.

Van Dyke, L., (2009), Projective Techniques, r2r Community (www.ksrinc.com/r2r/home.asp).

van Maanen, John (1988), Tales of the field. On writing ethnography, The University of Chicago Press, Chicago.

van Riel, C. B. M., N. E. Stroeker and O. J. M. Maathuis, (1998), "Measuring Corporate Images." Corporate Reputation Review, Vol. 1, pp. 313-326. 
Vince, R. and M. Broussine, (1996), "Paradox defense and attachment: accessing and working with emotions and relations underlying organizational change." Organization Studies, Vol. 17, No. 1, pp. 1-21.

Warren, S. (2009). Visual Methods in Organizational Research. The SAGE Handbook of Organizational Research Methods, SAGE, London.

Weick, K. E., (1979), The Social Psychology of Organizing, Addison-Wesley, Reading, MA.

Wheeldon, J. P. (2011), "Is a Picture Worth a Thousand Words? Using Mind Maps to Facilitate Participant Recall in Qualitative Research." The Qualitative Report, Vol. 16, No. 2, pp. 509-522.

Wheeldon, J. P. and Faubert, J. (2009), "Framing Experience: Concept Maps, Mind Maps, and Data Collection in Qualitative Research." International Journal of Qualitative Methods, Vol. 8, No. 3, pp. 68-83.

Wheeldon, J., and Ahlberg, M. K. (2012). Visualizing Social Science Research: Maps, Methods, \& Meaning, SAGE, London.

Whyte, J., Ewenstein, B. Hales M. and Tidd, J. (2008), "Visualizing Knowledge in ProjectBased Work." Long Range Planning, Vol. 41, No. 1, pp. 74-92.

Zuboff, S. (1988), In the Age of the Smart Machine: the Future of Work and Power, Basic Books, US. 
Figures and Tables

FIGURE 1

Strategy sketch used as 'ice-breaker' and reference in interview situations

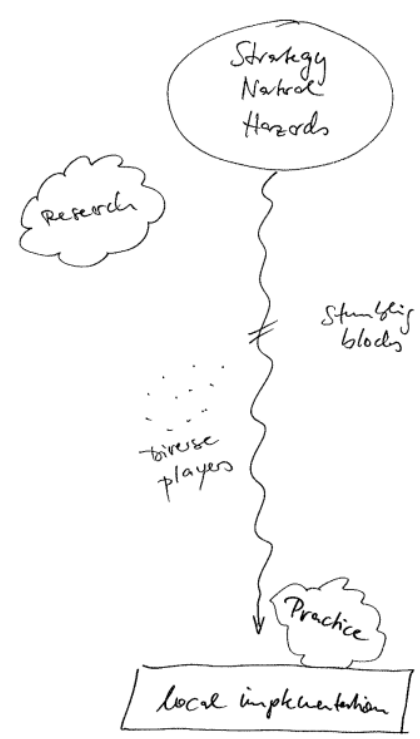

FIGURE 2

A visual timeline loaded on interactive visualization software, used as a template in face-toface interviewing

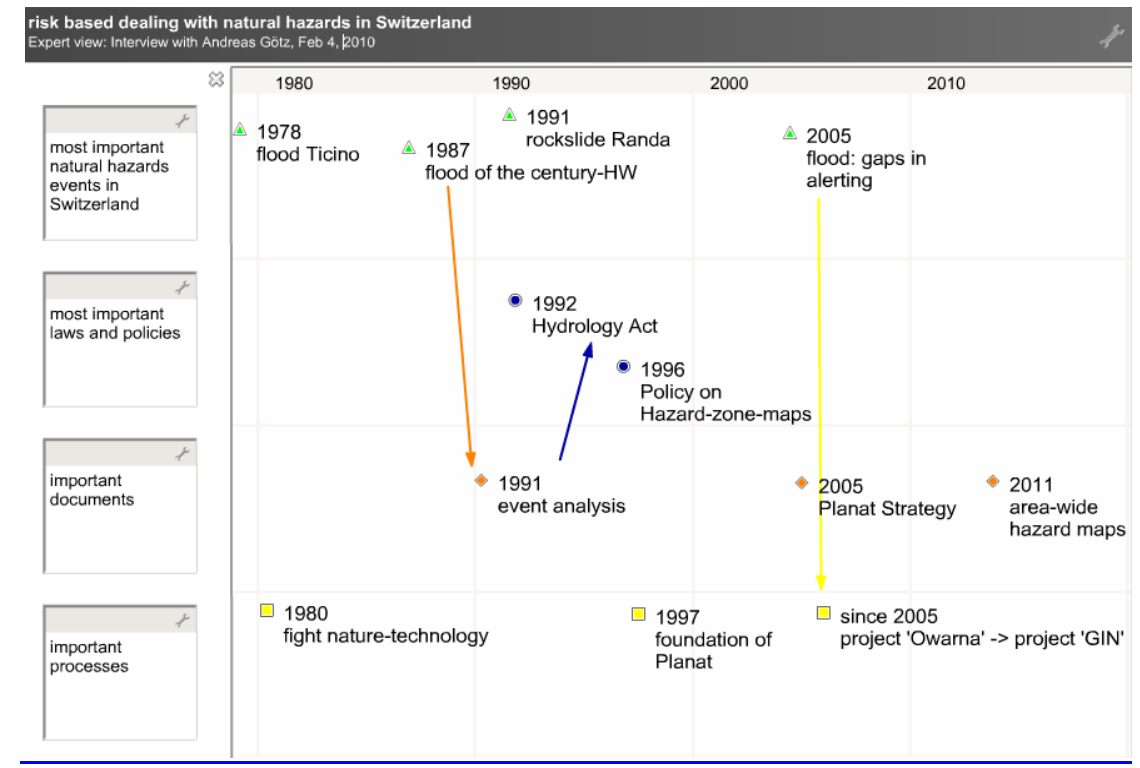


FIGURE 3

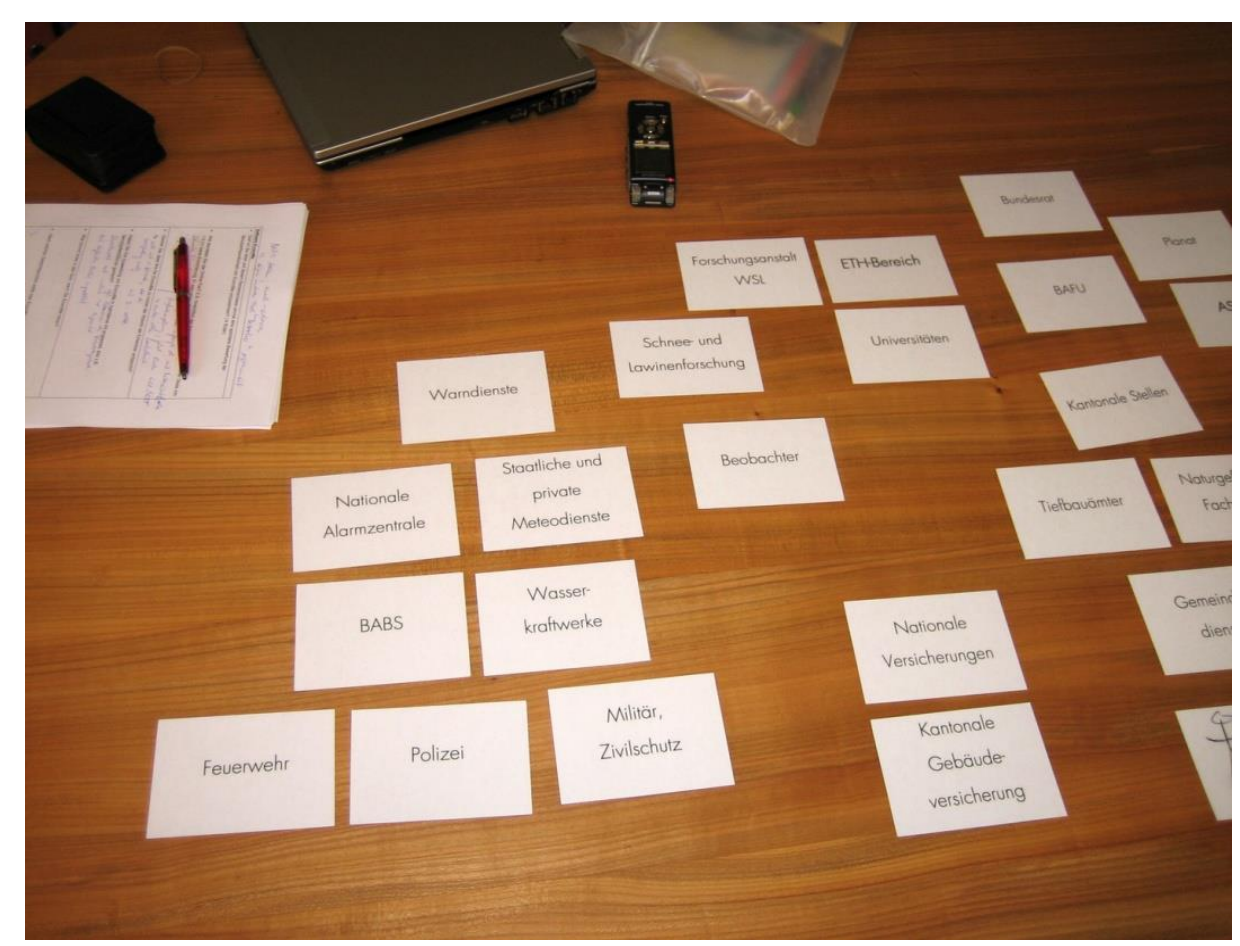


FIGURE 4

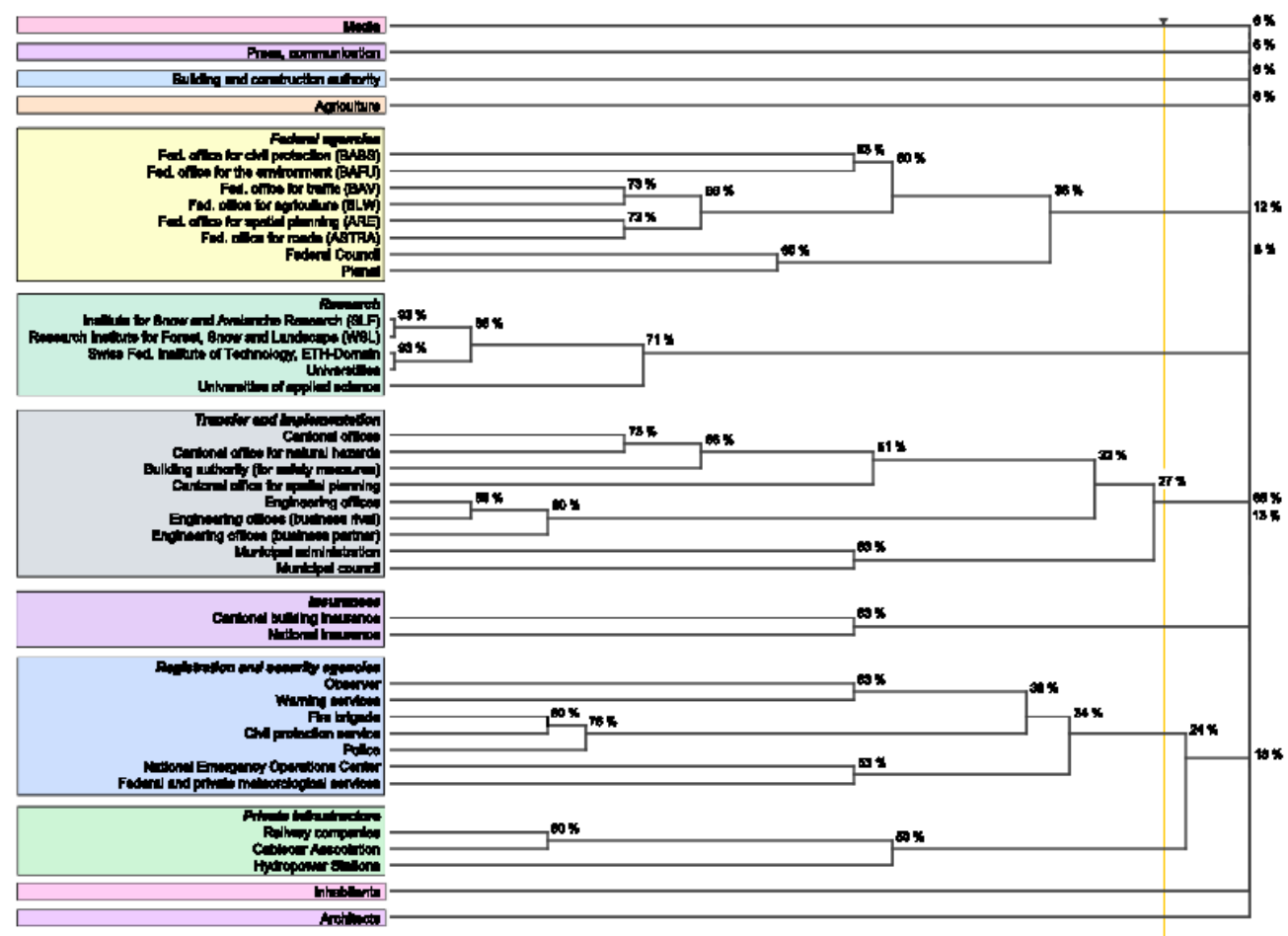

FIGURE 5

A completed visual template for focus group facilitation

visual templates (software- and poster-based)

no visual templates (flipchart)

dis ensure focus on the task at hand

require effort to develop a structure

d) ensure coverage of relevant themes

involve the risk to go off-topic

d) facilitate build-up of common ground

make difficult to build on each other

d. make easier to see interconnections

(e.g., complementarities)

53 jump-in effect

d) 'think out of the box' effect

59 'trapped in the box' effect

d) meeting effectiveness is less dependent on facilitator's ability

meeting effectiveness is largely dependent on facilitator's ability

d) ease the facilitation task (e.g., the facilitator has more time to listen)

d) revisability

no revisability

Q1: What are the advantages / disadvantages of the different group support systems? 
TABLE 1

A practical approach for integrating visual projection and facilitation

Interview Stage and Challenges Suggested Visualization Technique and Corresponding Advantages

Initiating the interview:

Visual Projection: The interviewee is presented

In the initial stage of the interview, with a visual stimulus and engaged in an associathe researcher should build rapport, and motivate the interviewee tion, expression, completion, or construction exercise.

to speak freely and to give exhaustive answers.

Projective exercises with visual stimuli are engaging and motivating for most interviewees. By removing respondents' inhibitions, visual projection enables the interviewer to elicit insights, perceptions and feelings. Moreover, visual stimuli increase recall of past experiences, thereby leading to more copious data.

In structured and semi-structured interviews, visual stimuli can be used also to clarify the scope and sequence of the interview, and therefore function as a signpost to set the stage for the conversation.

Conducting the interview:

The interviewer is faced with the dual challenge to guide the conversation while carefully processing what is being said. At the same time, the interviewee is confronted with cognitive challenges such as ordering thoughts and sustaining attention. Furthermore, knowledge asymmetries between the interviewer and the interviewee may
Visual Facilitation: The interviewer completes visual templates by prompting answers from the interviewees.

Visual facilitation fosters attention, reflection, and deliberation, while keeping focus on the interview questions. The simultaneous completion of visual templates enables both the interviewer and the interviewee to keep track of the conversation, thereby creating a collective memory. Because the responses remain visible, the interviewees can easily 
disrupt the interview flow.

In focus groups, the challenges of qualitative interviewing are further exacerbated by the threats of conformity pressure, interpersonal conflict, and unbalanced participation. build on them and provide corrections if needed.

In turn, the collaborative construction of visual templates, together with the intuitiveness of the visual language provides mechanisms for spanning knowledge boundaries between the interviewer and the interviewee.

In focus groups, visual facilitation instigates and maintains interaction and fosters balanced participation by providing ground rules for turntaking. The depersonalization effect brought by visual templates mitigates the risk of interpersonal conflict and lead interviewees to speak freely.

Wrapping-up the interview:

Before closing the interview, the researcher should identify preliminary results, and establish confidence in the data collected.

Visual Facilitation: The interviewer summarizes the main points that emerged in the interview by discussing the completed templates with the interviewee. The template's contents are thus re-injected as visual stimuli into the interview process.

The review of visual templates enables the interviewer and the interviewee to clarify misunderstandings. At the same time, the interviewer can gather further insights and probe answers.

Analysing the data: Visual Data Analysis: The integration of visual daPurely verbal interviews may lead ta reported on multiple templates allows for a quick to highly idiosyncratic data, and in turn make it difficult to identify exploration of common and unique themes emerged from the interviews. If necessary, the researcher can patterns. Moreover, sociolinguistic assumptions may bias the analysis and interpretation of findings. explore group differences by comparing the visual templates filled out by different categories of participants. An aggregate visualization can then be produced to summarize and present main findings to research participants for the purposes of data validation.

The integration of visual data (collected via projec- 
tion and facilitation techniques) and verbal data

(i.e., transcripts of interviewees' accounts) provides

multiple sources of evidence, and hence strengthens

the validity.

\section{ENDNOTES}

[1] An earlier version of this article was presented at the 2012 Annual Meeting of the Academy of Management in Boston (Research Methods Division), and at the 2011 International Conference on Information Visualization in London (Knowledge Visualization track).

[2] The examples stem from two PhD research projects: the first project (knowledge communication in a community of practice) and all qualitative one-to-one interviews were conducted by Nicole Bischof (Swiss National Science Foundation project no. CR12I1_126917). The second project (knowledge sharing in inter-organizational collaboration) and the related focus group interviews were conducted by Alice Comi during her appointment at the University of Lugano, Switzerland (Università della Svizzera italiana). Both PhD research projects were supervised by Martin J. Eppler.

\section{ACKNOWLEDGMENTS}

The project on knowledge communication in a community of practice - discussed in the section "Examples from the Field" - was funded by the Swiss National Science Foundation (project no. CR12I1_126917, applicants Nicole Bischof and Martin Eppler).

The authors would like to acknowledge Dr Susan Ainsworth, QROM Associate Editor, and two anonymous reviewers for their valuable comments on an earlier version of this paper. This paper also benefited from conversation with Sabrina Bresciani, Friederike Hoffmann, and members of the Knowledge Communication Research Group (http://www.knowledgecommunication.org/). 